\title{
DIABETES INSIPIDUS COMO MANIFESTAÇÃO INICIAL DE LEUCEMIA MIELOIDE AGUDA EM PACIENTE COM MONOSSOMIA DO CROMOSSOMO 7
}

\author{
DIABETES INSIPIDUS AS INITIAL MANIFESTATION OF ACUTE MYELOID \\ LEUKEMIA IN A PATIENT WITH MONOSOMY OF CHROMOSOME 7
}

\author{
Amanda Dias Lima Morais ${ }^{1}$, Adriana Girardi ${ }^{2}$, Mariluce Riegel ${ }^{3,4}$, \\ Alini Vargas ${ }^{5}$, Tatiana Helena Rech ${ }^{1}$
}

\begin{abstract}
RESUMO
O diabetes insipidus (DI) central é uma síndrome caracterizada pela incapacidade de concentração urinária devido à deficiência do hormônio antidiurético. O envolvimento do sistema nervoso central é frequente nas leucemias, mas a ocorrência de DI é rara e confere pior prognóstico. A patogênese do DI na leucemia não é totalmente conhecida, mas a infiltração do eixo hipotálamo-hipofisário por células leucêmicas parece ser um fator responsável. O presente relato descreve o caso de um paciente que apresentou DI como primeira manifestação de leucemia mieloide aguda e que evoluiu com dificuldades de ajustes do sódio sérico, da poliúria e da reposição volêmica, necessitando de permanência prolongada em unidade de cuidados intensivos.
\end{abstract}

Palavras-chave: diabetes insipidus; leucemia mieloide aguda; monossomia; cromossomo 7

\section{ABSTRACT}

Central diabetes insipidus (DI) is a syndrome characterized by the inability to concentrate urine due to a lack of antidiuretic hormone. Involvement of the central nervous system is common in acute leukemia, but the occurrence of DI is rare and determines a worse prognosis. The pathogenesis of DI in leukemia has not been fully understood yet, but infiltration of the hypothalamic-pituitary axis by leukemic cells seems to be involved. This report describes a case of a patient who presented with $\mathrm{DI}$ as the first manifestation of acute myeloid leukemia. Difficulties in the management of serum sodium, fluid replacement and polyuria led to prolonged length of stay in an intensive care unit.

Keywords: diabetes insipidus; acute myeloid leukemia; monosomy; chromosome 7

O diabetes insipidus (DI) caracteriza-se pela incapacidade de concentração urinária que leva à excreção de volumes anormalmente elevados de urina diluída devido à diminuição da reabsorção da água nos ductos coletores renais. No DI central, existe diminuição dos níveis de hormônio antidiurético $(A D H)$, enquanto no DI nefrogênico existe resistência à ação do hormônio em nível renal ${ }^{1}$.

O envolvimento pituitário ocorre em $0,6 \%$ dos casos de leucemia mieloide aguda (LMA $)^{2-5}$. Este relato descreve o caso de um paciente internado no centro de terapia intensiva (CTI) do Hospital de Clínicas de Porto Alegre (HCPA) cuja primeira manifestação da doença hematológica foi DI. Além disso, discute as dificuldades do manejo hidroeletrolítico encontrado nesse paciente.
Clin Biomed Res. 2017;37(1):55-58

1 Serviço de Medicina Intensiva, Hospital de Clínicas de Porto Alegre (HCPA). Porto Alegre, RS, Brasil.

2 Departamento de Medicina Interna, Hospital de Clínicas de Porto Alegre (HCPA). Porto Alegre, RS, Brasil.

3 Serviço de Genética Médica, Hospital de Clínicas de Porto Alegre (HCPA). Porto Alegre, RS, Brasil.

4 Programa de Pós-graduação em Genética e Biologia Molecular, Universidade Federal do Rio Grande do Sul (UFRGS). Porto Alegre, RS, Brasil.

5 Serviço de Hematologia, Hospital de Clínicas de Porto Alegre (HCPA). Porto Alegre, RS, Brasil.

Autor correspondente:

Tatiana Helena Rech threch@hcpa.edu.br Hospital de Clínicas de Porto Alegre (HCPA)

Rua Ramiro Barcelos, 2350

90035-903, Porto Alegre, RS, Brasil. 


\section{CASO CLÍNICO}

D. P. C. C., do sexo masculino, 66 anos, branco, foi encaminhado ao HCPA para tratamento de LMA. $\mathrm{Na}$ admissão, apresentava 206 mil leucócitos, com $74 \%$ de blastos no sangue periférico e com função renal e eletrólitos normais. A biópsia de medula óssea confirmou o diagnóstico de LMA subtipagem M4 (mielomonocítica). A análise do cariótipo encontra-se na Figura 1.

Em menos de 24 horas da internação, o paciente evoluiu com hipoxemia, sendo transferido ao CTI. Foi iniciada leucoferese de urgência para tratamento de síndrome de leucoestase. A despeito da redução de $50 \%$ na contagem de leucócitos e melhora do quadro respiratório, o paciente apresentou confusão mental e aumento progressivo do sódio sérico, com hipernatremia máxima atingindo $161 \mathrm{mEq} / \mathrm{L}$, associada à polidipsia e à poliúria de 7 litros em 24h. Foi levantada hipótese diagnóstica de DI, confirmada pela presença de osmolaridade urinária de $143 \mathrm{mOsm} / \mathrm{L}$, densidade urinária de 1004 e sódio urinário de $28 \mathrm{mmol} / \mathrm{L}$. O teste terapêutico com acetato de desmopressina (DDAVP) levou ao diagnóstico de DI de origem central, promovendo diminuição importante da diurese e aumento da densidade e osmoloridade urinárias. A tomografia computadorizada de crânio não evidenciou lesões em hipófise.

Após a estabilização do quadro clínico, o paciente recebeu alta do CTI. Nessa ocasião, o próprio paciente informou que vinha bebendo muita água e "não saia de perto da torneira". Em menos de $24 \mathrm{~h}$ após a alta do CTI, apresentou novo episódio de diminuição da consciência e instabilidade hemodinâmica, sendo reinternado no CTI. Apresentava-se hipotenso, hipoperfundido, hipovolêmico e oligúrico. Após reposição volêmica vigorosa, voltou a apresentar poliúria e hipernatremia responsivas a DDAVP. Foi calculado déficit de água livre de 5 litros, repostos ao longo de $24 \mathrm{~h}$ com solução salina $0,45 \%$. Seguiu-se uma grande dificuldade de ajustes do sódio sérico e da reposição volêmica e desenvolvimento de insuficiência renal aguda. A possibilidade de DI nefrogênico associado foi considerada, em virtude da dificuldade de manejo hidroeletrolítico e da perda da resposta inicial ao DDAVP. Porém, o paciente evoluiu com anúria e

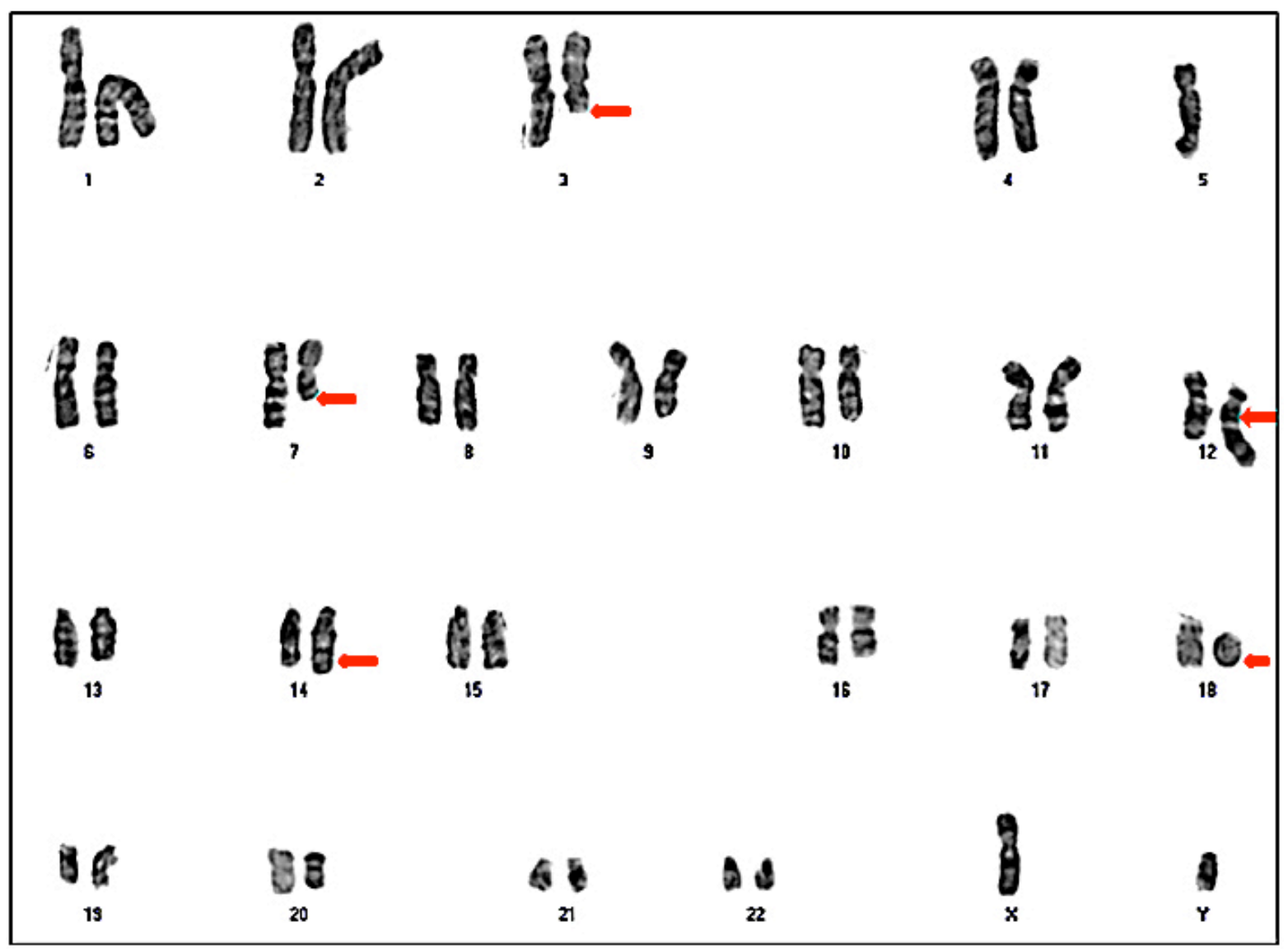

Figura 1: Cariótipo masculino por meio de banda GTG (banda G pelo método tripsina-Giemsa) mostrando clone neoplásico portador de monossomia pacial do cromossomo 7 ou del(7q) e rearranjo cromossômico complexo envolvendo os cromossomos 3, 5, 12, 14 e 18 (setas em vermelho). 
necessidade de terapia de substituição renal, não sendo possível a confirmação desse diagnóstico. O acúmulo de disfunções orgânicas e a não resposta à quimioterapia resultaram no óbito do paciente após 28 dias de cuidados intensivos.

\section{DISCUSSÃO}

Neste relato, apresentamos o caso de um paciente cuja manifestação inicial rara de LMA foi DI central, que passou a ser sintomática quando foi internado no CTI e deixou de ter acesso irrestrito à água.

Na LMA, o DI pode preceder a doença, ser concomitante ou ser a primeira manifestação de recaída ${ }^{5}$. O DI cursa com aumento de mortalidade em pacientes com $\mathrm{LMA}^{6,7}$. O tratamento do $\mathrm{DI}$ é difícil e exige monitorização seriada da natremia sérica, a fim de evitar complicações da correção rápida do sódio. $\mathrm{O}$ tratamento medicamentoso do DI central baseia-se na utilização de DDAVP. A forma de administração e as doses devem ser tituladas de acordo com a gravidade do DI. A forma intranasal costuma ser a primeira escolha, mas, em pacientes com quadro de hipernatremia grave e poliúria intensa, como o caso relatado, o uso intravenoso é recomendado ${ }^{8}$. A instituição do tratamento quimioterápico em paciente com LMA pode normalizar os níveis hormonais e resolver o DI, o que não aconteceu no paciente em questão, sugerindo a destruição irreversível das células produtoras de $\mathrm{ADH}^{3,9}$. No presente caso, foram necessárias doses crescentes de DDAVP, mesmo em vigência de insuficiência renal aguda, o que levantou a hipótese de DI nefrogênico associado. O tratamento do DI nefrogênico é feito basicamente com dieta restrita em sódio e diuréticos tiazídicos ${ }^{10}$. A avaliação da resposta ao tratamento de DI nefrogênico no nosso paciente não foi possível devido à evolução para falência renal aguda e necessidade de diálise.

A patogênese do DI na leucemia não é clara. Postula-se que a infiltração do eixo hipotálamo-hipofisário por células leucêmicas seja um dos fatores responsáveis ${ }^{3,11}$, mas essa é uma explicação parcial, já que muitos doentes com leucemia têm infiltração tumoral da região hipotalâmico-hipofisária e não desenvolvem $\mathrm{Dl}^{2,9}$. Fatores genéticos associados à monossomia do cromossomo 7 , como a del(7q), ou alterações no cromossomo 3 parecem estar associados ao desenvolvimento do $\mathrm{Dl}^{2}$. O mecanismo proposto para explicar a associação da del(7q) é a expressão anormal do gene responsável pela tradução da proteína GP130, uma glicoproteína da superfície dos granulócitos cuja desregulação predispõe ao $\mathrm{DI}^{12}$. Adicionalmente à monossomia dos cromossomos 5 e 7 [del(7q)] observada em nosso paciente, o cariótipo mostrou anormalidades provavelmente secundárias e não específicas originadas de rearranjos cromossômicos complexos (figura 1). A associação entre DI e deleção do cromossomo 7 , apesar de rara, está bem estabelecida na literatura ${ }^{2}$. Porém, a apresentação combinada de diversas alterações cromossômicas associadas à del $(7 q)$ da forma como este paciente apresentava não estão descritas na literatura até o momento, tornando o caso ainda mais raro.

Em conclusão, o aparecimento de confusão mental e hipernatremia em paciente com LMA deve alertar quanto à presença de DI, que pode se tornar evidente apenas no momento em que o paciente deixa de ter acesso livre à água. O não reconhecimento da síndrome pode ter consequências graves e o manejo pode tornar-se um desafio, além de poder ser a única indicação de permanência do paciente sob cuidados intensivos.

\section{REFERÊNCIAS}

1. Robertson GL. Diabetes insipidus. Endocrinol Metab Clin North Am. 1995;24:549-72.

2. Lavabre-Bertrand $T$, Bourquard $P$, Chiesa J, Berthéas MF, Lefort G, Taïb $J$, et al. Diabetes insipidus revealing acute myelogenous leukaemia with a high platelet count, monosomy 7 and abnormalities of chromosome 3: a new entity? Eur J Haematol. 2001;66:66-9.

3. Philippakos D, Kakouros S, Dervenoulas J, Pontidas E. Diabetes insipidus as a complication of acute myelomonocytic leukaemia. Postgrad Med J. 1983;59:93-4.
4. Harb A, Tan W, Wilding GE, Battiwalla M, Sait SN, Wang ES, et al. Acute myeloid leukemia and diabetes insipidus with monosomy 7. Cancer Genet Cytogenet. 2009;190:97-100.

5. Miller VI, Campbell WG JR. Diabetes insipidus as a complication of leukemia: a case report with a literature reviews. Cancer. 1971;28:666-73.

6. Porcu P, Cripe LD, Ng EW, Bhatia $\mathrm{S}$, Danielson CM, Orazi A, et al. Hyperleukocytic leukemias and leukostasis: a review of pathophysiology, clinical presentation and management. Leuk Lymphoma. 2000;39:1-18.

7. Frangoul HA, Shaw DW, Hawkins D, Park J. Diabetes insipidus as a presenting symptom of acute myelogenous leukemia. J Pediatr Hematol Oncol. 2000;22:457-9.

8. Vande Walle J, Stockner M, Raes A, Nørgaard JP. Desmopressin 30 years in clinical use: a safety review. Curr Drug Saf. 2007;2:232-8.

9. Ra'anani P, Shpilberg O, Berezin $M$, Ben-Bassat I. Acute leukemia relapse presenting as central diabetes insipidus. Cancer. 1994;73:2312-6. 
10. Bockenhauer D, Bichet DG.

Pathophysiology, diagnosis and management of nephrogenic diabetes insipidus. Nat Rev Nephrol. 2015;11:576-88.
11. Pagano L, Voso MT, Sica S, Leone G. Recovery from diabetes insipidus associated with AML after a BMT conditioning regimen including busulfan. Bone Marrow Transplant. 1993;11:175-6.
12. de la Chapelle A, Lahtinen R. Monosomy 7 predisposes to diabetes insipidus in leukaemia and myelodysplastic syndrome. Eur J Haematol. 1987;39:404-11.

Recebido: Ago 05, 2016

Aceito: Jan 27, 2017 